\title{
Model-based cardiac diagnosis of Pulmonary Embolism
}

\author{
C. Starfinger ${ }^{1}$, C.E. Hann ${ }^{1}$, J.G. Chase ${ }^{1}$, T. Desaive ${ }^{2}$ \\ A. Ghuysen ${ }^{3}$, G.M. Shaw ${ }^{4}$ \\ ${ }^{1}$ Centre of Bioengineering, University of Canterbury, Christchurch, New Zealand \\ ${ }^{2}$ Institute of Physics, University of Liège, Belgium \\ ${ }^{3}$ Hemodynamics Research Laboratory, University of Liège, Belgium \\ ${ }^{4}$ Department of Intensive Care Medicine, Christchurch Hospital, Christchurch, New Zealand
}

August 3, 2006

\section{Abstract}

A minimal cardiac model has been shown to accurately capture a wide range of cardiovascular system dynamics commonly seen in the intensive care unit (ICU). However, standard parameter identification methods for this model are highly non-linear and non-convex, hindering real-time clinical application. An integral-based identification method that transforms the problem into a linear, convex problem, has been previously developed, but was only applied on continuous simulated data with random noise. This paper extends the method to handle discrete sets of clinical data, unmodelled dynamics, a 
significantly reduced data set thta requires only the minimum and maximum values of the pressure in the aorta, pulmonary artery and the volumes in the ventricles. The importance of integrals in the formulation for noise reduction is illustrated by demonstrating instability in the identification using simple derivative-based approaches. The CVS model and parameter identification method are then clinically validated on porcine data for pulmonary embolism. Errors for the identified model are within $10 \%$ when re-simulated and compared to clinical data. All identified parameter trends match clinically expected changes. This work represents the first clinical validation of these models, methods and approach to cardiovascular diagnosis in critical care.

Keywords: cardiovascular system, cardiac model, parameter identification, integral method, pulmonary embolism

\section{Introduction}

Cardiac disease state is highly patient specific and difficult to accurately diagnose due to the limited measurements available. In addition, the body's natural reflex responses try to restore circulatory equilibrium, which can often mask the underlying symptoms $[1,2]$. Successful diagnosis and treatment 
often rely on the experience and intuition of clinical staff. Thus, a physiological, identifiable and validated computer model offers several potential advantages in diagnosis and therapy selection, by aggregating diverse patient data into a compact, patient specific, clinically relevant and potentially real-time assessment of circulatory status.

There are many CVS models in the literature ranging from very complex finite element models [3-6] to relatively simpler pressure volume approaches [7-9]. However, the focus is often on only specific areas of CVS dysfunction. Although there are full CVS models, patient-specific parameter optimization is either not considered or restricted to small subsets of the overall much larger parameter set (e.g.[10, 11]). This restriction to specific CVS aspects can dramatically limit the range of CVS disturbances that can be detected, thus prohibiting use as a broader diagnostic tool for patients with unknown condition. For relatively larger, more complex system models computational cost and feasibility can also become a major issue.

This research employs a physiologically validated minimal model $[12-$ 15] capable of capturing patient dynamics commonly seen in an ICU, while using a relatively small number of physiological variables. A highly efficient solution method [16] provides the necessary simplicity, flexibility and rapid 
forward simulation that is required in a clinical environment. An integralbased parameter identification method has been also been developed and shown, in simulation, to rapidly and accurately identify virtually the entire parameter set in the presence of significant measurement noise [17]. However, a relatively large measured data set was assumed, including continuously measured pressure and flow waveforms. Such measurements might not always be clinically available.

In this paper, the integral method is extended to allow discrete sets of clinical data and is shown to be robust to unmodelled dynamics and measurement noise. The measurements utilized are also reduced from prior work to a more clinically feasible set. The use of integrals in the formulation is shown to be critical for stability, even with locally smoothed curves, as compared to numerical derivative-based identification approaches. The method is initially tested on simulations of pulmonary embolism that capture all the physiologically expected responses. The CVS model and integral method are then clinically validated on a porcine model of pulmonary embolism. 


\section{Methodology}

\section{$2.1 \quad$ CVS model}

The CVS model is a lumped parameter model [18], where the left and right ventricle chambers are characterized by the flow in and out of the chamber, the pressure up- and downstream and the resistances of the valves, and inertia of the blood. An overview of the model is given in Figure 1. To add flexibility and better match waveform shape as well as peak values, the model is extended from [18] to allow a slightly non-linear pressure volume relationship in the aorta and pulmonary artery. The equations for the left ventricle are defined:

$$
\begin{aligned}
V_{p c d} & =V_{l v}+V_{r v} \\
P_{p c d} & =P_{0 p c d} \cdot\left(e^{\lambda_{p c d(}\left(V_{p c d}-V_{0 p c d}\right)}-1\right) \\
P_{p e r i} & =P_{p c d}+P_{t h} \\
V_{l v f} & =V_{l v}-V_{s p t} \\
P_{l v f} & =d r i L \cdot E_{e s l v f} \cdot\left(V_{l v f}-V_{d l v f}\right) \\
& +(1-d r i L) \cdot P_{0 l v f} \cdot\left(e^{\lambda_{l v f\left(V_{l v f}-V_{0 l v f}\right)}}-1\right)
\end{aligned}
$$




$$
\begin{gathered}
P_{l v}=P_{l v f}+P_{p e r i} \\
P_{p u}=E_{p u} \cdot\left(V_{p u}-V_{d p u}\right)+P_{t h} \\
\dot{V}_{a o}=Q_{a v}-Q_{s y s} \\
Q_{s y s}=\frac{P_{a o}-P_{v c}}{R_{s y s}} \\
P_{a o}=E_{a o} \cdot\left(V_{a o}-V_{d a o}\right)^{f} \\
\dot{V}_{l v}=Q_{a v}-Q_{m t} \\
\dot{Q}_{m t}=H\left(H\left(P_{p u}-P_{l v}\right)+H\left(Q_{m t}\right)\right) \cdot \frac{\left(P_{p u}-P_{l v}-R_{m t} \cdot Q_{m t}\right)}{L_{m t}} \\
\dot{Q}_{a v}=H\left(H\left(P_{l v}-P_{a o}\right)+H\left(Q_{a v}\right)\right) \cdot \frac{\left(P_{l v}-P_{a o}-R_{a v} \cdot Q_{a v}\right)}{L_{a v}}
\end{gathered}
$$

where $H$ is the Heaviside function, $f$ is a nonlinear factor ranging from 0.8 to 1.4, and all other variables are as shown in Figure 1. Similar equations are used for the right ventricle and pulmonary/ systemic circulation. For a more detailed description see $[12-14,16,18]$. The parameter $f$ in Equation (10) provides more flexibility to capture the shape and peak of $P_{a o}$ seen in clinical data.

\subsubsection{Activation Function}

The electrical activation of the left and right ventricles are described using a driver function and time varying elastance to model cardiac muscle activation 
$[7,18]$. For clinical validation on the porcine data, separate driver functions are chosen for the left and right ventricles.

$$
\begin{aligned}
d r i_{L} & =A_{L} \cdot e^{\left(-b_{L} \cdot\left(t-\frac{\text { period }}{c_{L}}\right)^{2}\right)} \\
d r i_{R} & =A_{R} \cdot e^{\left(-b_{R} \cdot\left(t-\frac{\text { period }}{c_{R}}\right)^{4}\right)} \\
\text { period } & =\frac{1}{\text { heartrate }}
\end{aligned}
$$

where $A_{L}=1, b_{L}=2582.177, c_{L}=2.07$ and $A_{R}=1, b_{R}=91.5975$, $c_{R}=2.18$ for the left $(L)$ and right ventricles $(R)$. The drivers are shown in Figure 2 for a period of $0.53 \mathrm{~s}$, and are developed from scaling pressures for the porcine data.

The use of two different driver functions is physiologically justified, as the electric signal spreads differently in both ventricles. More specifically, the cardiac activation pattern and times have been clinically observed to differ between the right and left ventricles $[19,20]$. The activation function is also defined to change as a function of the heart period. For human simulations the same driver function is used for both ventricles and the septum volume, and is defined $[7,18]$ :

$$
d r i=1 \cdot e^{\left(-80 \cdot\left(t-\frac{\text { period }}{2}\right)^{2}\right)}
$$




\subsubsection{Ventricular Interaction}

Ventricular interaction is an important dynamic $[21,22]$ and is included in the model. The septum volume is described by a time-varying $\mathrm{P}-\mathrm{V}$ relationship defined $[7,14,18]$ :

$$
P_{s p t}=d r i S \cdot E_{\text {esspt }}\left(V_{s p t}-V_{d s p t}\right)+(1-d r i S) \cdot P_{0 s p t}\left(e^{\lambda_{s p t}\left(V_{s p t}-V_{o s p t}\right)}-1\right)
$$

where the driver function $d r i S$ describing the activation of the septum, is

taken from $[7,14,18]$. The septum volume $V_{s p t}$ can be determined analytically using the methods in [17].

$$
V_{s p t}=a / b
$$

with $a$ and $b$ defined:

$$
\begin{aligned}
a & =\left(d r i S \cdot E_{e s s p t} \cdot V_{d s p t}+d r i L \cdot E_{e s l v f} \cdot V_{l v}-d r i R \cdot E_{e s r v f} \cdot V_{r v}\right. \\
& -(1-d r i S) \cdot P_{0 s p t} \cdot\left(b_{s p t} e^{-\lambda_{s p t} V_{o s p t}}-1\right)+(1-d r i L) \cdot P_{0 l v f} \cdot\left(b_{l v f} e^{\lambda_{l v f} V_{l v}}-1\right) \\
& \left.-(1-d r i R) \cdot P_{0 r v f} \cdot\left(b_{r v f} e^{\lambda_{r v f} V_{r v}}-1\right)\right)
\end{aligned}
$$




$$
\begin{aligned}
b & =\left(d r i S \cdot E_{\text {esspt }}-d r i L \cdot E_{e s l v f}-d r i R \cdot E_{\text {esrvf }}\right. \\
& +(1-d r i S) \cdot P_{0 s p t} \cdot a_{s p t} e^{-\lambda_{s p t} V_{o s p t}}-(1-d r i L) \cdot P_{0 l v f} \cdot a_{l v f} e^{\lambda_{l v f} V_{l v}} \\
& \left.+(1-d r i R) \cdot P_{0 r v f} \cdot a_{r v f} e^{\lambda_{r v f} V_{r v}}\right)
\end{aligned}
$$

where $a_{s p t}, a_{l v f}, a_{r v f}, b_{s p t}, b_{l v f}, b_{r v f}$ are defined:

$$
\begin{aligned}
x_{1} & =V_{s p t, o l d}+\Delta V_{s p t} ; x_{2}=V_{s p t, o l d}-\Delta V_{s p t} \\
a_{s p t} & =\frac{e^{\lambda_{s p t} x_{2}}-e^{\lambda_{s p t} x_{1}}}{x_{2}-x_{1}} ; a_{l v f}=\frac{e^{\lambda_{l v f} x_{2}}-e^{\lambda_{l v f} x_{1}}}{x_{2}-x_{1}} ; a_{r v f}=\frac{e^{\lambda_{r v f} x_{2}}-e^{\lambda_{r v f} x_{1}}}{x_{2}-x_{1}} \\
b_{s p t} & =e^{\lambda_{s p t} x_{1}}-\left(e^{\lambda_{s p t} x_{2}}-\frac{e^{\lambda_{s p t} x_{1}}}{x_{2}-x_{1}} x_{1}\right) \\
b_{l v f} & =e^{\lambda_{l v f} x_{1}}-\left(e^{\lambda_{l v f} x_{2}}-\frac{e^{\lambda_{l v f} x_{1}}}{x_{2}-x_{1}} x_{1}\right) \\
b_{r v f} & =e^{\lambda_{r v f} x_{1}}-\left(e^{\lambda_{r v f} x_{2}}-\frac{e^{\lambda_{r v f} x_{1}}}{x_{2}-x_{1}} x_{1}\right)
\end{aligned}
$$

and $V_{s p t, o l d}$ is the $V_{s p t}$ in the previous time step and $\triangle V_{s p t}=0.1 \mathrm{ml}$. Note

that for the case of simulations of human $d r i L=d r i R=d r i S$.

\subsubsection{Reflex actions (human simulations)}

The effect of CVS diseases on the cardiovascular system can be significantly altered by the compensation from nervous system reflex mechanisms. Thus, reflex actions are included in the CVS model for the pulmonary embolism 
simulation. It is assumed that vasoconstriction is proportional to a drop in pulmonary artery pressure $\left(P_{a o}\right)$ and is modeled by increasing the systemic vascular resistance $\left(R_{\text {sys }}\right)$ by $34 \%$ for a drop in average $P_{a o}$ from $100 \mathrm{mmHg}$ to $80 \mathrm{mmHg}$. Other reflex mechanisms include venous constriction, increased heart rate (HR) and increased ventricular contractility $[1,19]$. Their activation is also assumed to be proportional to the drop in the average pressure in the aorta $\left(P_{a o}\right)$. The proportionality constants are estimated based on clinically observed CVS hemodynamic responses reported in the literature [23-25]. More specifically, HR and ventricular contractility are increased by 80 to 120 beats per minute and $67 \%$ whereas the venous dead space $V_{d v c}$ is decreased by $35 \%$ respectively for a drop in average Pao to $80 \mathrm{mmHg}$. Figure 3 shows how $R_{s y s}$ is varied as a function of $\triangle P_{a o}$.

\subsection{Integral-Based Parameter Identification}

To uniquely determine the parameters, the model equations are transformed using integrals. A previously designed integral-based parameter identification method [17] is extended in this paper to rapidly identify the patient specific parameters from limited discrete data. The assumed measured data is the discrete minimum and maximum values of the pressure 
in the aorta $\left(P_{a o, \max }, P_{a o, \min }\right)$, pulmonary artery $\left(P_{p a, \max }, P_{p a, \min }\right)$, and the discrete maximum and minimum volumes of the left and right ventricles $\left(V_{l v, \text { max }}, V_{l v, \text { min }}, V_{r v, \max }, V_{r v, \text { min }}\right)$. Hence, unlike prior work [17], no waveforms are required and there are $60 \%$ less measurements ( 4 total) required in this approach.

\subsubsection{Scaling model outputs - discrete data}

For discrete data, the waveforms are not known, therefore the integral method of [17] cannot be directly applied. However, waveforms can be artificially generated by scaling a set of previously calculated model outputs to best fit the maximum and minimum measured data values for the pressures and volumes. The assumption is that these validated model waveforms are reasonably conformable with the actual clinical case. In return, significantly less measurement and potentially fewer invasive catheters are required.

The scaled signal, Sig $_{n e w}$, is obtained from a previously calculated signal, Sig $g_{\text {old }}$, as follows: 


$$
\begin{aligned}
\text { Sig }_{\text {new }} & =a \cdot S i g_{\text {old }}+b \\
a & =\frac{\left(S i g_{m, \text { max }}-S i g_{m, \text { min }}\right)}{\left(\text { Sig }_{s, \text { max }}-S i g_{s, \text { min }}\right)} \\
b & =\frac{\left(S i g_{s, \text { max }} \cdot S i g_{m, \text { min }}-S i g_{m, \text { max }} \cdot S i g_{s, \text { min }}\right)}{\left(S i g_{s, \text { max }}-S i g_{s, \text { min }}\right)}
\end{aligned}
$$

where the subscript $s$ refers to simulated output and the subscript $m$ refers to measured data. For example, Figure 4 shows the pressure in the aorta $\left(P_{a o}\right)$ after scaling with $a=0.6832$ and $b=2.417$, with the measured maximum and minimum values denoted by a circle.

\subsubsection{Scaling model outputs - porcine data}

For the porcine data continuous waveforms are measured in $V_{l v}, V_{r v}, P_{a o}$ and $P_{p a}$. However, the same scaling approach can be used to simplify the parameter identification. In particular, scaling effectively filters noise and unmodelled dynamics from the data. The identification problem is thus restricted to dynamics in the model. Note that the final comparison is still made to the original data and this approach is only done to minimize computational effort and complexity in the identification process. An example of scaling in this clinical porcine case is shown in Figure 5, for the pressure in the aorta $\left(P_{a o}\right)$ before and after scaling, with $a=0.5871$ and $b=6.7166$. 
However, matching only the maximum and minimum values has the limitation that the waveform shape may not be precisely captured. For the porcine data, better matches to the waveform shapes were obtained by introducing a slight non-linearity into the pressure volume relationship in the aorta and pulmonary artery, as defined by Equation (10). A range of $f$ parameters ranging from 0.8 to 1.4 were tested, where each time the integral method was applied. The $f$ value producing the best waveform match in the aorta and pulmonary artery was chosen. Hence, more complexity could be readily added to the model to better capture the observed dynamics with minimal effect on computational time.

\subsubsection{Integral identification problem formulation}

Consider the left ventricle defined in Equations (8) and (13). Assume that $Q_{a v}, Q_{m t}, P_{a o}, V_{l v}, V_{s p t}$ and $P_{p e r i}$ are either measured or estimated from measured data. Integrating Equation (11) from $t_{e b}$ to $t$ during ejection and from $t_{f b}$ to $t$ during filling gives an expression for $V_{l v}(t)[17]$ :

$$
\begin{aligned}
V_{l v}(t) & =V_{l v}\left(t_{e b}\right)-\int_{t_{e b}}^{t} Q_{a v}(t) d t, t_{e b} \leq t \leq t_{e f} \\
& =V_{l v}\left(t_{f b}\right)+\int_{t_{f b}}^{t} Q_{m t}(t) d t, t_{f b} \leq t \leq t_{f e}
\end{aligned}
$$


where $t_{e b}$ is the beginning of ejection, and $t_{f b}$ is the beginning of filling, $t_{e e}$ stands for end-ejection and $t_{f e}$ for end-filling respectively. For simplicity, $V_{d a o}=0$ and $f=1$ in the following equations.

Integrating Equation (8) from 0 to $t$, solving for $V_{a o}(t)$, and then using Equations (9) and (10) yields:

$$
\begin{aligned}
P_{a o}(t)= & E_{a o}\left(V_{a o}(0)+\int_{0}^{t} Q_{a v}(t) d t-\frac{1}{R_{s y s}} \int_{0}^{t} P_{a o}(t) d t\right. \\
& \left.-\frac{1}{R_{\text {sys }}} \int_{0}^{t} P_{v c}(t) d t\right)
\end{aligned}
$$

Under the assumption that $P_{v c}=P_{v c 0}$ is an unknown constant, Equation (32) can be rewritten:

$$
P_{a o}(t)=P_{a o 0}+E_{a o} \int_{0}^{t} Q_{a v}(t) d t+A_{1} \int_{0}^{t} P_{a o}(t) d t+A_{2} t
$$

where $A_{1}$ and $A_{2}$ are defined:

$$
A_{1}=-\frac{E_{a o}}{R_{s y s}}, A_{2}=\frac{E_{a o} P_{v c 0}}{R_{s y s}}
$$

The best linear least squares fit of Equation (34) to the measured pressure waveform $P_{a o}$ over one heart beat will determine $E_{a o}$ and $R_{s y s}$ over that 
heart beat. Similarly, given an approximation to $V_{s p t}$ and $P_{\text {peri }}$ in Equation (5), Equations (12) and (13) can be integrated across the filling and ejection stages respectively. A linear least-squares optimization can then be similarly used to determine $R_{a v}, R_{m t}, E_{\text {eslvf }}$ and $P_{0 l v f}$. The right ventricle can be treated similarly.

Given the pressure waveforms through the aorta and pulmonary artery, the flows into and out of the left and right ventricles, as well as their volumes, a system of linear equations can thus be defined for the full CVS model [17]:

$$
\begin{aligned}
& A \cdot \vec{x}=\vec{b} \\
& \vec{x}=\left(L_{a v}, L_{m t}, L_{t c}, L_{p v}, E_{e s l v f}, P_{0 l v f}, E_{e s r v f}, P_{0 r v f}, E_{a o}, E_{p a}, E_{v c}, E_{p u}\right. \\
& \left.\quad R_{a v}, R_{m t}, R_{t c}, R_{p v}, P_{a o 0}, P_{p u 0}, P_{p a 0}, P_{v c 0}, R_{s y s}, R_{p u l}\right)^{T}
\end{aligned}
$$

with $\vec{x}$ being the solution vector of the parameters to be identified, which can be found by linear least squares. More details about this integral method and parameter definitions can be found in [17].

Ventricular interaction is also included, however the volume of the septum is not known and not directly measurable in an ICU. As an initial approximation, this volume is set to zero. The resulting parameters identified by 
the integral method are then used to resimulate the model and produce an approximation of the septum volume $\left(V_{s p t}\right)$. The parameter identification is then run a second time using this $V_{s p t}$ value, producing a modified set of identified parameters that better account for ventricular interaction.

In this research, the parameters are identified for each period of measured data during the porcine experiment of pulmonary embolism. Thus, time varying changes from the initial healthy state to the fully diseased state are captured, as might be desired for a clinical system. Hence, model identification can provide a potential means of monitoring CVS disease state in the highly dynamic critical care patient.

\subsubsection{Simulation using optimized parameters}

Figure 6 shows the overall process of the simulation and parameter identification algorithm. After the porcine specific parameters have been identified for a respective point in time, these parameters are then used to rerun the model simulation. The simulated output is then compared to the clinical data. However, due to errors in the initial approximations of $V_{s p t}$ and the unmeasured flows $\left(Q_{a v}, Q_{m t}, Q_{p v}, Q_{t c}\right)$, and the process of scaling output signals, parameter identification should be iterated to ensure optimal convergence. 
Fast convergence consistently occurred within 3-5 iterations in this study and is stopped when the relative error between model output and clinical data reaches a set tolerance.

\section{Results \& Discussion}

\subsection{Simulated Pulmonary Embolism in Human}

The CVS model and previously identified human parameters [12-14, 17] are used to generate simulated pulmonary embolism data. Pulmonary embolism is caused by a blood clot obstructing the pulmonary circulation and is simulated by increasing the pulmonary resistance $R_{\text {pul }}$ by $30 \%$ every 50 heart beats for a total of 300 heart beats. This gives an overall increase in $R_{p u l}$ of $150 \%$. To account for measurement noise, white gaussian noise of $5 \%$ and $10 \%$ is added to the (simulated) measurements for pressure in the aorta, pulmonary artery and both ventricle volumes.

During pulmonary embolism, blood is backing up in the right ventricle due to increased afterload. The overfilled right ventricle compresses the underfilled left ventricle and thus, the right ventricle expansion index (RVEDV/LVEDV) increases [26, 27]. Figure 7 shows the true expansion in- 
dex versus the re-simulated expansion indexes obtained using the identified parameter sets from the simulated pulmonary embolism experiment.

The left panels of Figure 8 show the simulated pressure in the left ventricle $\left(P_{l v}\right)$, the left ventricle volume $\left(V_{l v}\right)$ and the pressure in the aorta $\left(P_{a o}\right)$. In each case, $10 \%$ Gaussian white noise is added to the pressure and volume signals, except for the ventricular pressure (top panel) as it is not measured or used in the analysis. Only the maximum and minimum values of $P_{a o}$ and $V_{l v}$ in the noise corrupted signals are used to identify the system parameters. The dotted lines in each panel are the re-simulated signals generated with the identified parameter set. Similar results for the right ventricle are given in the right panel. Although the pressure in left and right ventricles is not known and not used during the parameter identification process, the re-simulated data matches it very well.

Finally, Figure 9 shows the identified pulmonary resistance over the 300 heart beats. Increased pulmonary resistance is the hallmark of pulmonary embolism. Here, it is consistently detected with up to $10 \%$ random noise added.

The pressure-volume relationship for the left ventricle is shown in Figure 10 for 6 different points in time during the pulmonary embolism experiment 
with $10 \%$ measurement noise. The CVS identification method produces parameters, which when re-simulated match the clinical data very closely with average errors of $3.18 \pm 1.79 \mathrm{mmHg}(7.20 \%)$ and $3.81 \pm 3.38 \mathrm{ml}(4.67 \%)$ for the maximum and minimum pressures and volumes respectively. These identification results are summarized in Table 1, which also shows an accurately captured rise in pulmonary resistance.

\subsection{Integral vs Derivative identification approaches}

Rather than formulating Equations (1) - (13) in terms of integrals [17] a potentially simpler way is to directly substitute the measured or estimated data into Equations (1) - (13). This approach would require differentiating the signals for Equations (8), (11), (12) and (13). For the case of scaled signals, the local noise is effectively removed and the signals are smooth suggesting that differentiation may be suitable. A similar system of linear equations to Equations (35) and (36) would be obtained without the initial conditions $P_{a 00}, P_{p a 0}, P_{v c 0}$ and $P_{p u 0}$ which are essentially integration constants.

However, although local measurement noise is removed there is still modelling error that occurs from scaling the signals. Specifically, a scaled waveform can only match the maximum and minimum values of a measured signal 
and will not necessarily have the same waveform shape. Figure 11 shows an example where the scaled and measured pressure and volume waveforms are superimposed. Note that "measured" in this case refers to the model generated signal using pre-selected parameters that represent a "virtual" patient.

Figure 12 shows a comparison of the derivatives $\frac{d P_{a o}}{d t}, \frac{d P_{p a}}{d t}$ and integrals $\int_{0}^{t} P_{a o} d t, \int_{0}^{t} P_{p a} d t$ for the scaled versus true signals. A similar comparison is made between $\frac{d V_{V v}}{d t}, \frac{d V_{r v}}{d t}$ and $\int_{0}^{t} V_{l v} d t, \int_{0}^{t} V_{r v} d t$ in Figure 13. Very large errors can be seen in the differentiated signals, thus showing how differentiation amplifies the modelling error between the curves in Figure 11, even though the signals are locally smooth. In contrast, integration effectively reduces modelling error as shown by the upper panels in Figures 12 and 13. Figures 14 and 15 further demonstrate the differences by displaying the percentage errors for both methods, where the derivative-based approach shows an effective instability.

The parameters identified by the derivative-based method are then used to rerun the CVS model and produce pressure and volume curves, for comparison to the (simulated) measured data. This process is repeated in all the measured periods during the pulmonary embolism simulation experiment. The results in Figure 16 show significant large errors in the matching of the 
signals. The total mean error in the identified parameters, versus the true values simulated, over all periods was $419 \% \pm 1363 \%$. Thus, the integral formulation provides a more robust parameter identification in the presence of modelling or measurement error.

\subsection{Porcine Pulmonary Embolism}

Finally, the integral-based parameter identification is applied to clinical porcine data for a true clinical validation. The data was obtained from the Hemodynamics Research Laboratory, University of Liège, Belgium. In the experiments, a pig is injected with autologous blood clots every 2 hours to simulate pulmonary embolism [28]. Three pigs are presented for initial validation of the methods presented.

Figure 17 shows the simulated model output for the pressure in the left and right ventricles $\left(P_{l v s}, P_{r v s}\right)$, the volume in the left and right ventricles $\left(V_{l v s}, V_{r v s}\right)$ and the pressure in the aorta, pulmonary artery $\left(P_{a o s}, P_{p a s}\right)$ overlaid with the corresponding clinical data $\left(P_{l v p} / P_{r v p}, V_{l v p} / V_{r v p}, P_{a o p} / P_{p a p}\right)$ at 30 mins into the pulmonary embolism experiment of pig 1 . The simulation data matches the measured porcine data very well with errors within 2.36 $\mathrm{mmHg}(\sim 5.72 \%)$ and $1.47 \mathrm{ml}(\sim 2.16 \%)$ for the maximum and minimum 
pressures and volumes, respectively.

Figure 18 shows the Volume-Pressure waveforms for the left and right ventricles in more detail. The upper panel in Figure 18 is the simulated ventricle volume and the dotted line is the measured porcine volume for 2 heartbeats. The lower panel shows the same results obtained for the ventricle pressure. Finally, Figure 19 shows the resulting Pressure-Volume relationships (P-V loops) for the left and right ventricles. Errors in all cases are in the range of $0.15 \%$ to $4.76 \%$.

Figure 20 displays the $\mathrm{P}-\mathrm{V}$ loops for the left and right ventricle 120 minutes into the pulmonary embolism experiment. Although the model didn't exactly capture all the exact volume shapes in the left and right ventricles, the pressure waveform shapes were accurately captured, as well as the maximum and minimum pressures and volumes. The differences represent local, unmodelled dynamics, as might be expected. Overall, the errors in the maximum pressures and volumes that are typically used to define trends in different disease states are within $0.17 \%$ to $4.95 \%$, respectively.

Figure 21 displays the P-V loops at 180 minutes, which was the end of the experiment. Again the results show a very close match. Errors in the maximum pressures and volumes are all within $0.20 \%$ and $6.59 \%$, respectively. 
Figure 22 clearly shows that the identified subject (pig) specific parameters systemic and pulmonary vascular resistance, $R_{s y s}$ and $R_{p u l}$, differ significantly between healthy and disease state, with $R_{\text {pul }}$ increasing by $261.44 \%$. Furthermore, the model's ability to pick up reflex response can clearly be seen in Figure 22, as the pig increases systemic resistance to help restore blood pressure. However, near the end, when the pig is near death, systemic resistance $\left(R_{\text {sys }}\right)$ drops off. This last result is potentially a sign that the pig can no longer regulate hemodynamics effectively. The left and right ventricle contractilities $\left(E_{\text {eslvf }}, E_{\text {esrvf }}\right)$ also increased during the pulmonary embolization experiment. These contractilities are also known to be part of reflex response [29], providing some further confirmation of this result.

The derivative formulation of the parameter identification algorithm did not produce a parameter set that could be used to re-run the CVS simulation when using the clinical porcine data. This result further illustrates the instability and/or diffculty of this type of method for this problem.

\subsection{Summary of 3 pigs}

The results of the integral-based parameter identification method on the 3 pigs are summarized in Table 2. The mean and standard deviation of the 
absolute errors for the maximum and minimum pressure in aorta $\left(P_{a o}\right)$ and pulmonary artery $\left(P_{p a}\right)$, and left and right ventricle $\left(P_{l v}, P_{r v}\right)$ are given. The mean model response errors are within $2.21 \pm 2.15 \mathrm{mmHg}(\sim 5.52 \%)$ for the pressures and $2.37 \pm 2.01 \mathrm{ml}(\sim 3.49 \%)$ for the volumes. These results show that the minimal CVS model is able to capture the essential dynamics of the porcine CVS response to induced pulmonary embolism, over a selection of subjects.

\section{Conclusions}

The integral-based optimization successively identified patient specific parameters for the minimal cardiac model with inertial effects and ventricular interaction. A much reduced discrete set of measured data was employed compared to prior work. The use of integrals for identification of this model parameter identification, particularly in the presence of measurement noise and/or modelling error. In contrast, derivative-based methods failed to produce stable, reliable identification results. Thus, integrals are fundamental to handling both local measurement error [17] and modelling error in the parameter identification process.

Computationally, the parameter identification optimization problem is 
made linear and convex, where current approaches are non-linear and nonconvex. The results from clinical porcine data of pulmonary embolism show that clinically relevant and physiologically accurate parameter identification can be obtained to a clinical setting. These results will obviously need to be confirmed with further trials over broader sets of cardiac circulatory dysfunction. However, this integral approach has the potential to ensure medical staff can obtain rapid patient specific information to assist in diagnosis and therapy selection in clinical real time. 


\section{References}

[1] A. Grenvik, S. M. Ayres, P. R. Holbrook, and W. C. Shoemaker, editors. Textbook of Critical Care. W.B. Saunders Company, 2nd edition, 1989.

[2] A.C. Guyton and J.E. Hall. Textbook of medical physiology. Philadelphia: Saunders, 10th edition, 2000.

[3] C.S. Peskin and D.M. McQueen. Cardiac fluid dynamics. Rev.Biomed.Eng., 20 (5 6):451 459, 1992.

[4] D.M. McQueen, C.S. Peskin, and E.L. Yellin. Fluid dynamics of the mitral valve:physiological aspects of mathematical model. Am.J.Physiol., 242 (6):H1095 H1110, 19882.

[5] I.J. Legrice, P.J. Hunter, and B.H. Smaill. Laminar structure of the heart: mathematical model. Am.J.Physiol., 272:H2466H2476, 1997.

[6] P.J. Hunter and B.H. Smaill. Structure and function of the diastolic heart: material properties of passive myocardium, in: L.Glass,P.Hunter,A.McCulloch (Eds.), Theory of Heart. SpringerVerlag, Harrisonburg, 1991.

[7] D. C. Chung, S. C. Niranjan, J. W. Clark JR, A. Bidani, W. E. John- 
ston, J. B. Zwischenberger, and D. L. Traber. A dynamic model of ventricular interaction and pericardial influence. Am. J. Physiol., 272(6 Pt 2):H2942-2962, 1997.

[8] M. Ursino. A mathematical model of the carotid baroregulation in pulsating conditions. IEEE Trans.Biomed.Eng., 4:382 392, 1999.

[9] N. Stergiopulos, P. Segers, and N. Westerhof. Use of pulse pressure method for estimating total arterial compliance in vivo. Am.J.Physiol., 276 (2 Pt 2):H424 H428, 1999.

[10] J. T. Ottesen, M. S. Olufsen, and J. K. Larsen. Applied mathematical models in human physiology. Philadelphia : Society for Industrial and Applied Mathematics, 2004.

[11] R. Mukkamala and R. J. Cohen. A forward model-based validation of cardiovascular system identification. American Journal of Physiology. Heart and Circulatory Physiology, 281(6):H2714-2730, 2001.

[12] B. W. Smith. Minimal haemodynamic modelling of the heart \& circulation for clinical application. PhD thesis, University of Canterbury, 2004. 
[13] B.W. Smith, J.G. Chase, G.M. Shaw, and R.I. Nokes. Experimentally verified minimal cardiovascular system model for rapid diagnostic assistance. Control Engineering Practice, 13:1183-1193, 2005.

[14] B.W. Smith, J.G. Chase, G.M. Shaw, and R.I. Nokes. Simulating transient ventricular interaction using a minimal cardiovascular system model. Physiological measurement, 27:165-179, 2006.

[15] B.W. Smith, J.G. Chase, R.I. Nokes, G.M. Shaw, and G. Wake. Minimal haemodynamic system model including ventricular interaction and valve dynamics. Medical Engineering $\&$ Physics, 26:131-139, 2004.

[16] C. E. Hann, J. G. Chase, and G. M. Shaw. Efficient implementation of non-linear valve law and ventricular interaction dynamics in the minimal cardiac model. Computer Methods and Programs in Biomedicine, 80:65 $74,2005$.

[17] C. E. Hann, J. G. Chase, and G. M. Shaw. Integral-based identification of patient specific parameters for a minimal cardiac model. Computer Methods and Programs in Biomedicine, 81(2):181-192, 2006.

[18] B. W. Smith, J. G. Chase, R. I. Nokes, G. M. Shaw, and G. Wake. Minimal haemodynamic system model including ventricular interaction 
and valve dynamics. Medical Engineering \&3 Physics, 26(2):131-139, 2004.

[19] R. E. Klabunde. Cardiovascular Physiology Concepts. Lippincott Williams and Wilkins, 2004.

[20] C. Ramanathan, J. Ping, R. Ghanem, Ryu K., and Y. Rudy. Activation and repolarization of the normal human heart under complete physiological conditions. Proceedings of the National Academy of Sciences of America, 103 (16):6309-6314, April 18, 2006.

[21] K. T. Weber, J. S. Janicki, S. Shroff, and A. P. Fishman. Contractile mechanics and interaction of the right and left ventricles. Am. J. Cardiol., 47:686 695, 1981.

[22] M. A. Fogel, P. M. Weinberg, K. B. Gupta, J. Rychik, A. Hubbard, E. A. Hoffman, and J. Haselgrove. Mechanics of the single left ventricle: a study in ventricular-ventricular interaction ii. Circulation, 98:330 338, 1998.

[23] E. Braunwald. Heart Disease, A text book of cardiovascular medicine. 5th edition. W.B. Saunders Company, Philadelphia, 1997. 
[24] A. Despopoulos and S. Silbernagl. Color atlas of physiology. 5th edition. New York: Thieme, 2001.

[25] J.E. Parrillo and R.C. Bone. Critical care medicine, Principles of diagnosis and management. Mosby, St. Louis, Missouri, 1995.

[26] C.T. Gan, J.W. Lankhaar, J.T. Marcus, N. Westerhof, K.M. Marques, J.G. Bronzwaer, A. Boonstra, P.E. Postmus, and A. Vonk-Noordegraaf. Impaired left ventricular filling due to right-to-left ventricular interaction in patients with pulmonary arterial hypertension. Am J Physiol Heart Circ Physiol., 290(4):H1528-H1533, 2006, Epub 2005 Nov 11.

[27] J.C. Lualdi and S.Z. Goldhaber. Right ventricular dysfunction after acute pulmonary embolism: pathophysiologic factors, detection, and therapeutic implications. Am Heart J., 130(6):1276-1282, 1995.

[28] Th. Desaive, S. Dutron, B. Lambermont, P. Kolh, C. E. Hann, J. G. Chase, P.C. Dauby, and A. Ghuysen. Closed-loop model of the cardiovascular system including ventricular interaction and valve dynamics: application to pulmonary embolism. 12th Intl Conference on Biomedical Engineering (ICBME), Singapore, Dec 7-10 2005.

[29] D. Burkhoff and T. V. Tyberg. Why does pulmonary venous pressure 
rise after onset of LV dysfunction: a theoretical analysis. Am J Physiol Heart Circ Physiol, 265:H1819-H1828, 1993. 


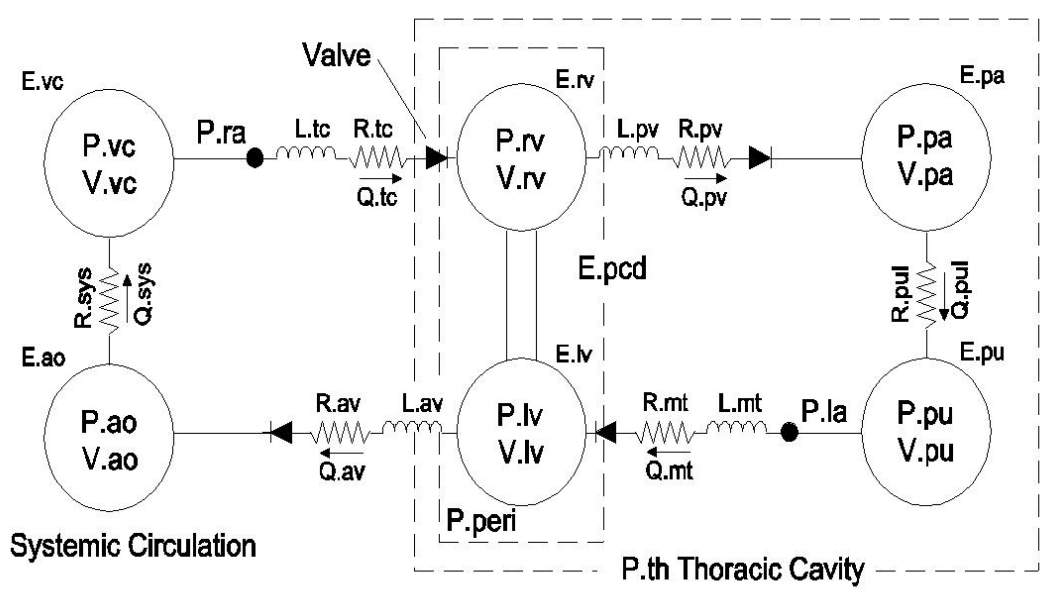

Figure 1: Minimal CVS model overview 


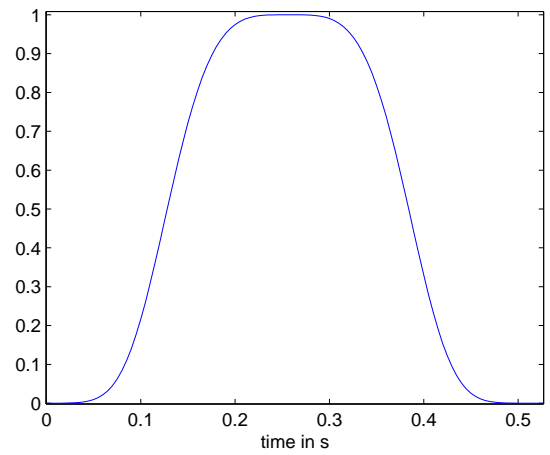

(a) Left ventricle

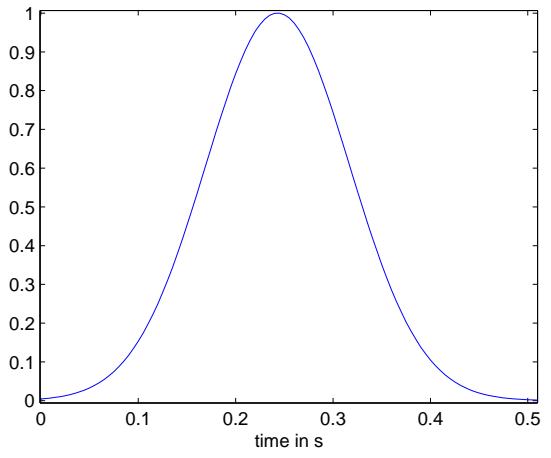

(b) Right ventricle

Figure 2: Driver functions for ventricle activation

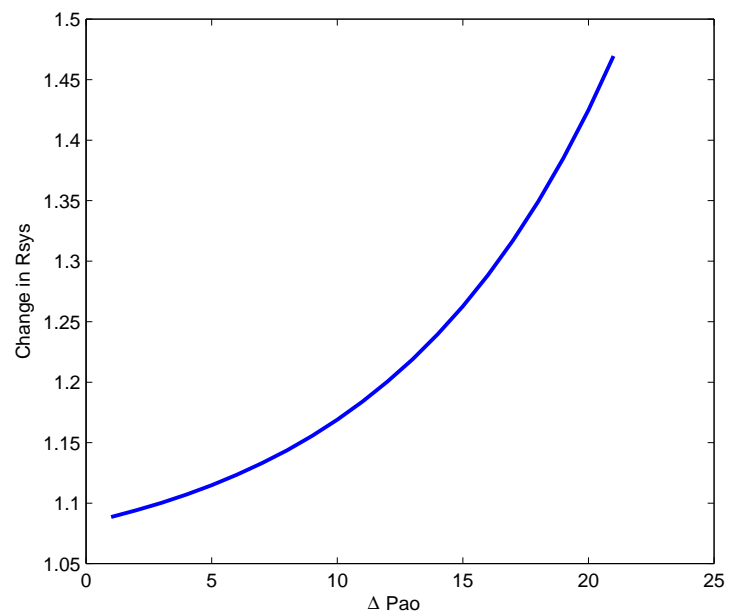

Figure 3: Varying $R_{\text {sys }}$ as a function of $\triangle P_{a o}$ 

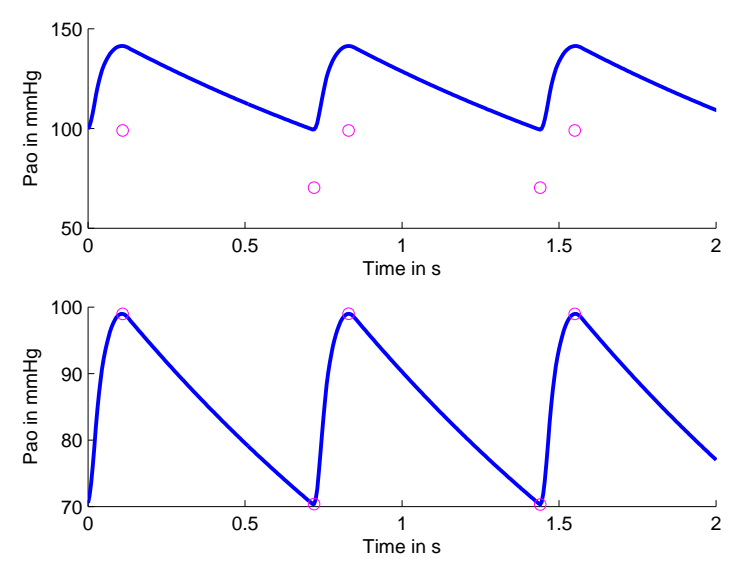

Figure 4: Simulated Pulmonary Embolism in Human: Pressure in aorta $\left(P_{a o}\right)$ before and after scaling
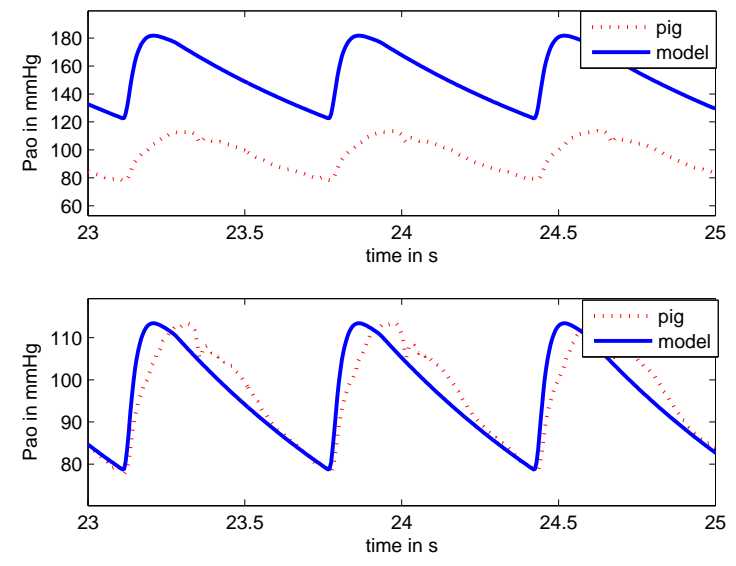

Figure 5: Porcine Pulmonary Embolism: Pressure in aorta $\left(P_{a o}\right)$ before and after scaling 


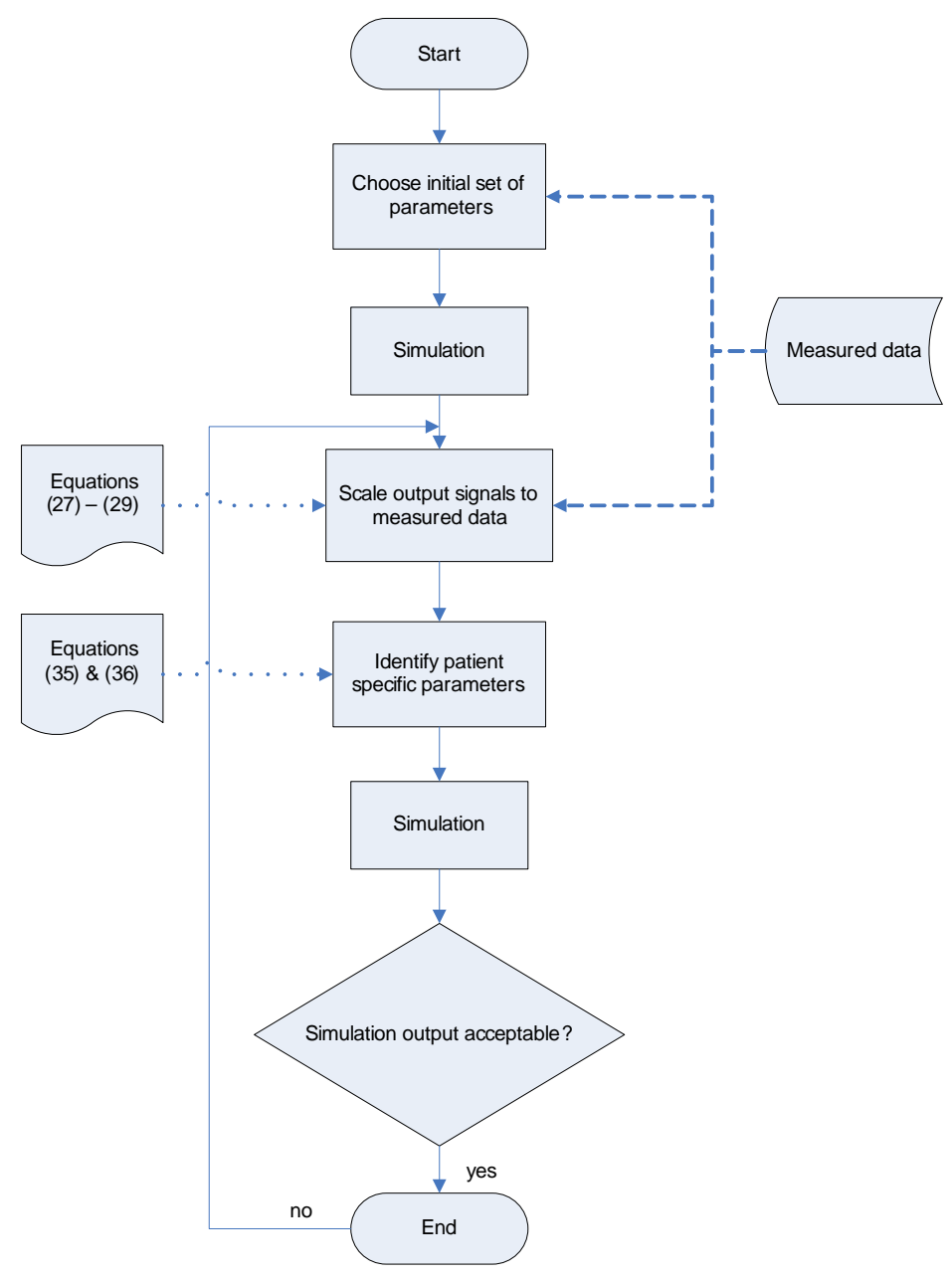

Figure 6: Flow chart of simulation and parameter identification algorithm 


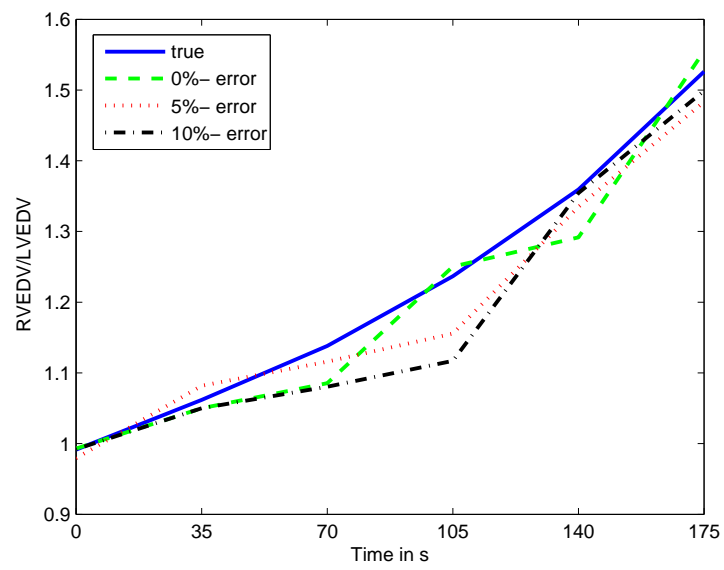

Figure 7: Right ventricle expansion index, RVEDV/LVEDV for 0,5 and 10\% measurement noise added for simulated human case
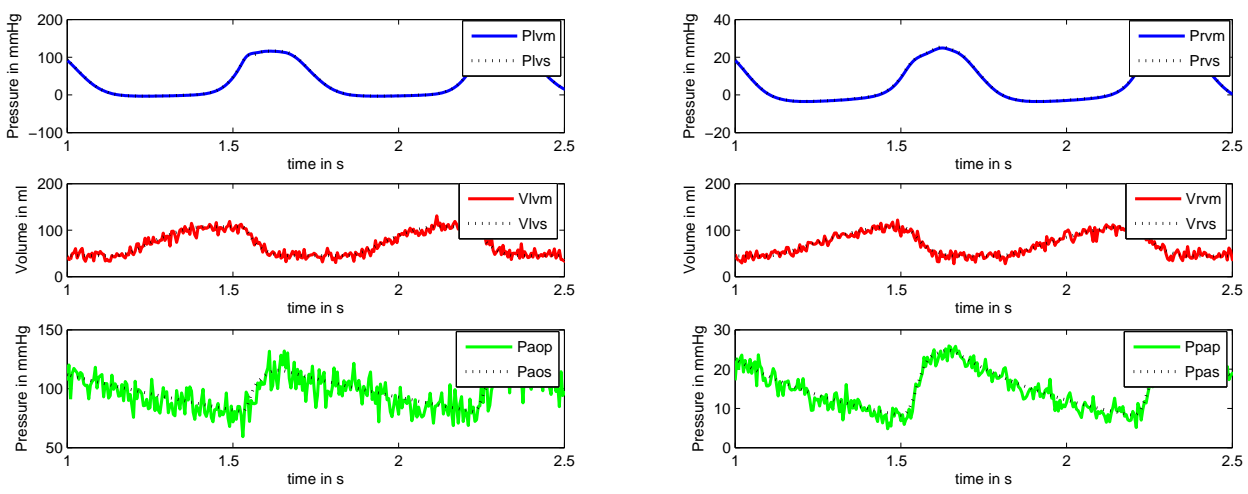

(a) Left Ventricle

(b) Right Ventricle

Figure 8: Integral-based identification (ID): pressures and volumes, measured (m) vs simulated (s) for simulated human case 


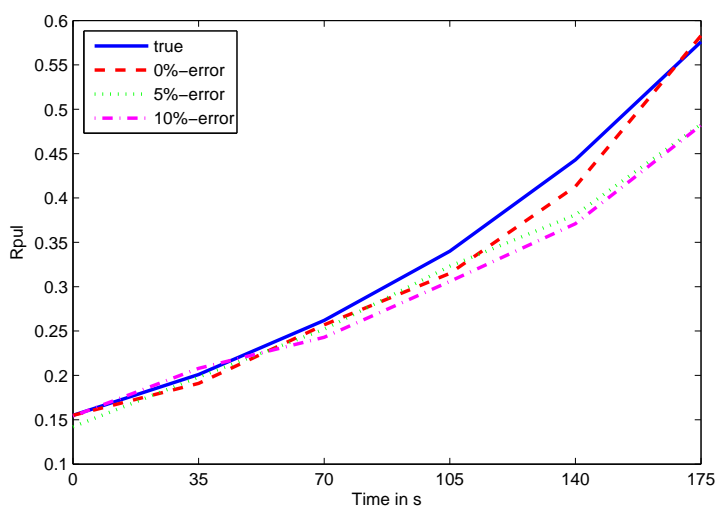

Figure 9: Identified pulmonary vascular resistance $\left(R_{\text {pul }}\right)$ for 0,5 and $10 \%$ measurement noise added for simulated human case 


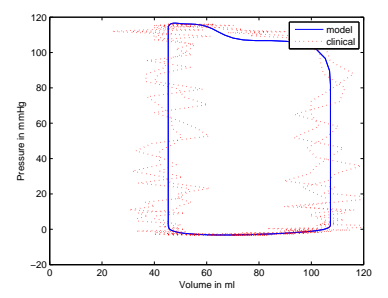

(a) time: $0 \mathrm{~s}$

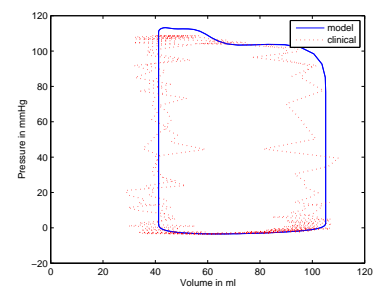

(d) time: $105 \mathrm{~s}$

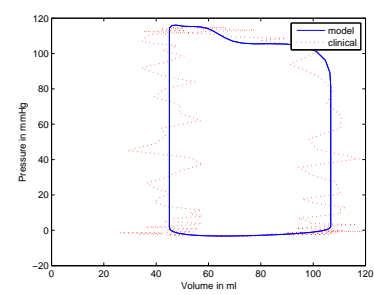

(b) time: $35 \mathrm{~s}$

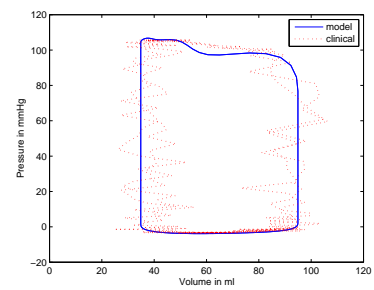

(e) time: $140 \mathrm{~s}$

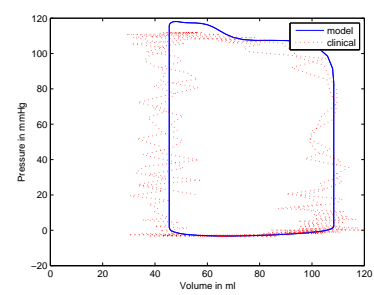

(c) time: $70 \mathrm{~s}$

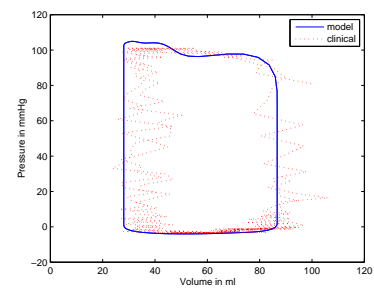

(f) time: $175 \mathrm{~s}$

Figure 10: Pressure-Volume Relationship for left ventricle during pulmonary embolism experiment and $10 \%$ measurement noise for simulated human case 

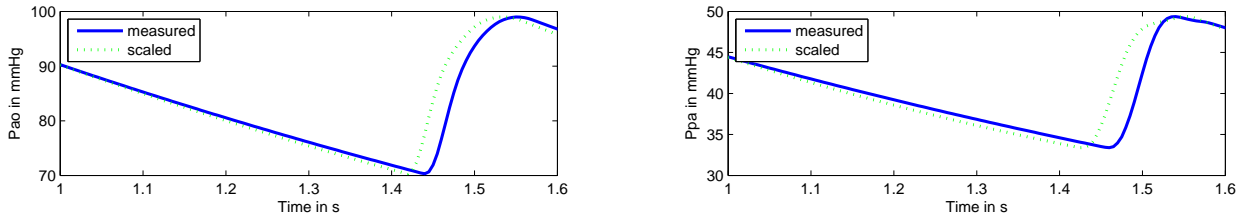

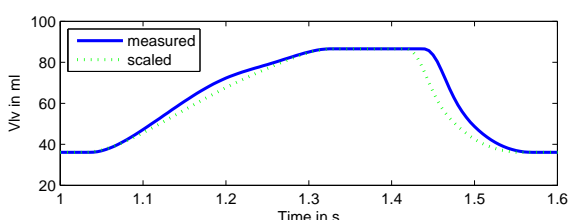

(a) Left ventricle

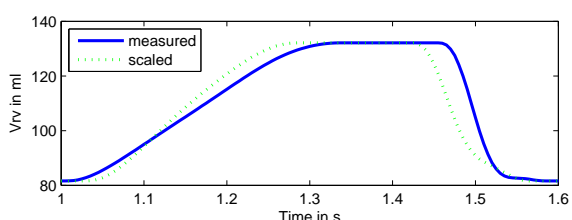

(b) Right ventricle

Figure 11: Comparison between measured (solid) and scaled (dashed) signal for simulated human case 

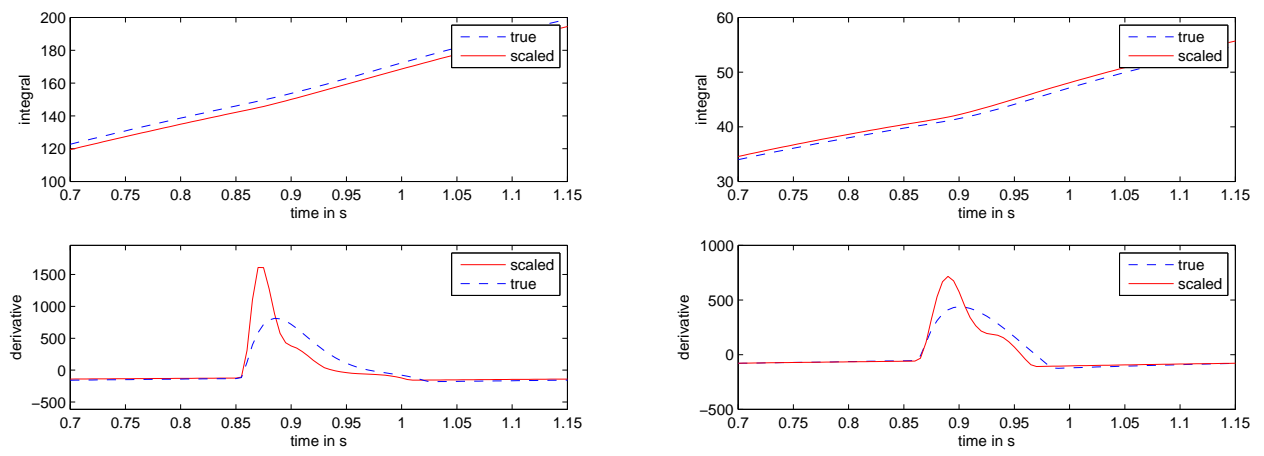

(a) Pressure in aorta, $P_{a o}$

(b) Pressure in pulmonary artery, $P_{p a}$

Figure 12: Comparison between integral and derivative for true (dashed) and scaled (solid) signal for simulated human case
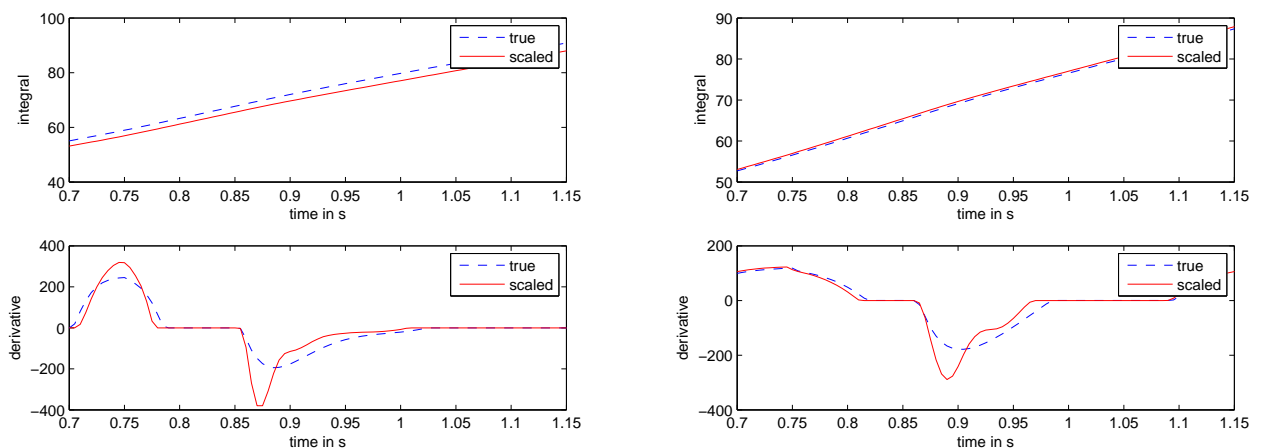

(a) Volume in left ventricle, $V_{l v}$

(b) Volume in right ventricle, $V_{r v}$

Figure 13: Comparison between integral and derivative for true (dashed) and scaled (solid) signal for simulated human case 

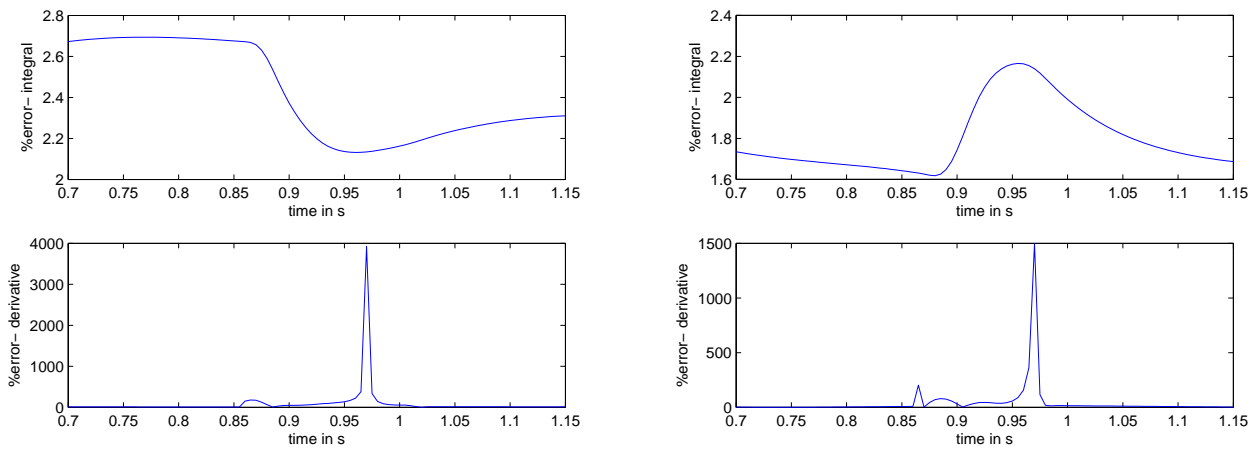

(a) Pressure in aorta, $P_{a o}$

(b) Pressure in pulmonary artery, $P_{p a}$

Figure 14: Percentage error for integral and derivative for simulated human case
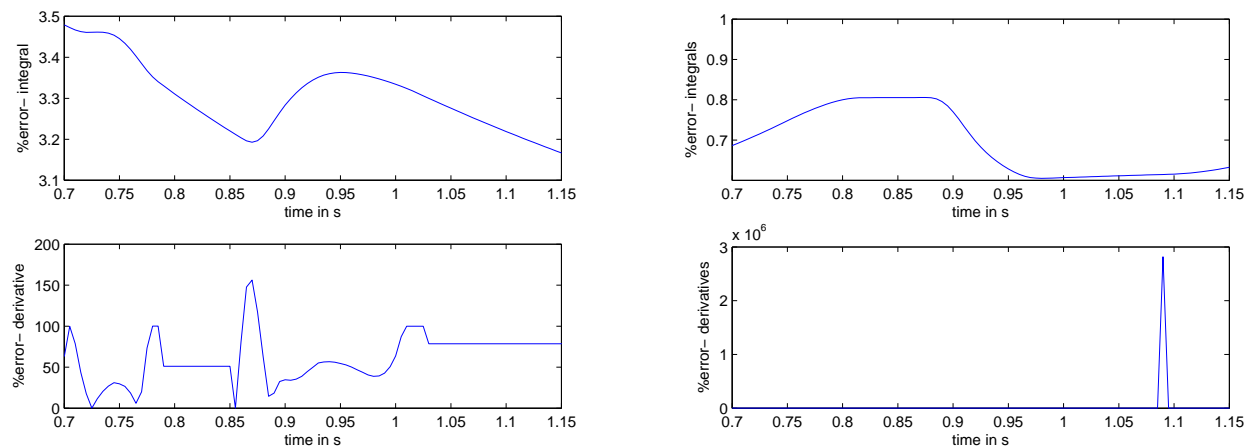

(a) Volume in left ventricle, $V_{l v}$

(b) Volume in right ventricle, $V_{r v}$

Figure 15: Percentage error for integral and derivative for simulated human case 

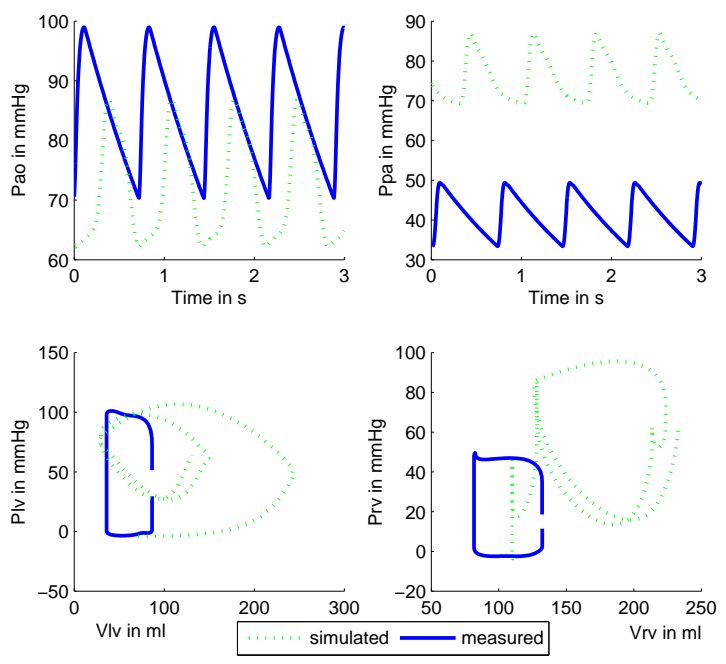

Figure 16: Derivative-based identification (ID): pressures and volumes, measured vs simulated, for a simulated human trial of pulmonary embolism 

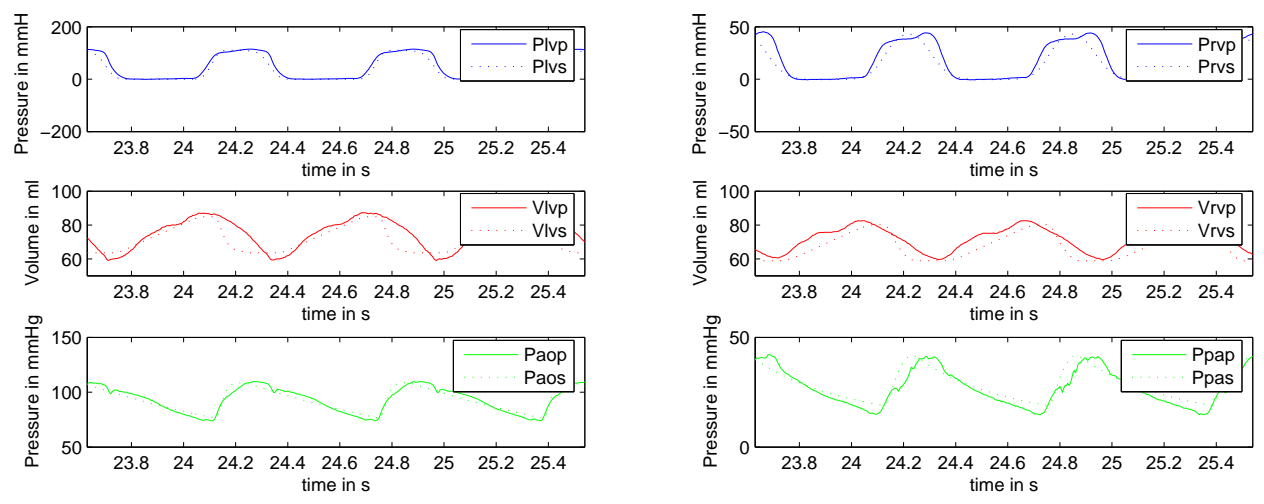

(a) 30 mins, left ventricle

(b) 30 mins, right ventricle

Figure 17: Porcine pulmonary embolism, pig 1, model output (s) vs clinical data (p) data
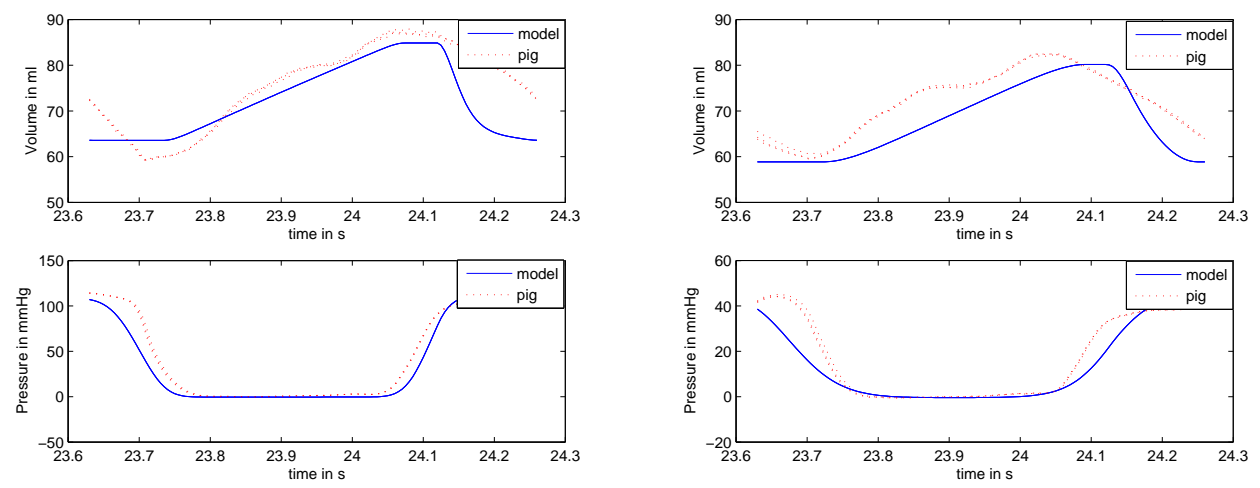

(a) 30 mins, left ventricle

(b) 30 mins, right ventricle

Figure 18: Porcine pulmonary embolism, pig 1, model output (s) vs clinical data (p) for Pressure and Volume 


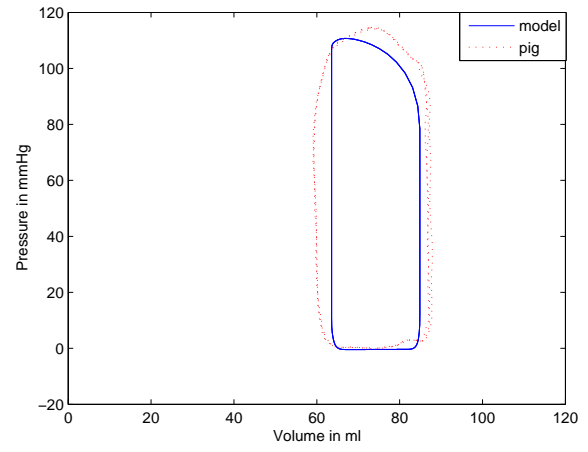

(a) 30 mins, left ventricle

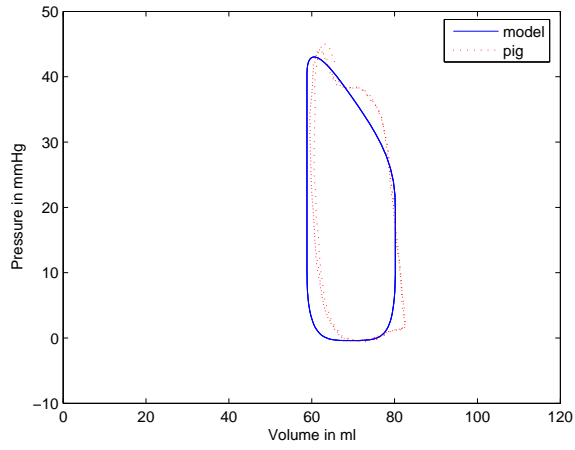

(b) 30 mins, right ventricle

Figure 19: Porcine pulmonary embolism, pig 1, Pressure-Volume Relationship

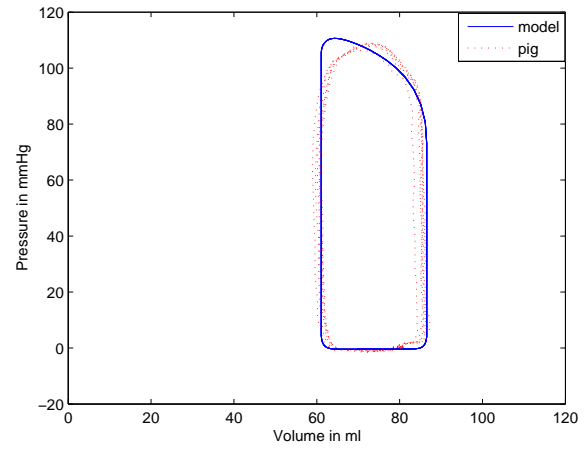

(a) 120 mins, left ventricle

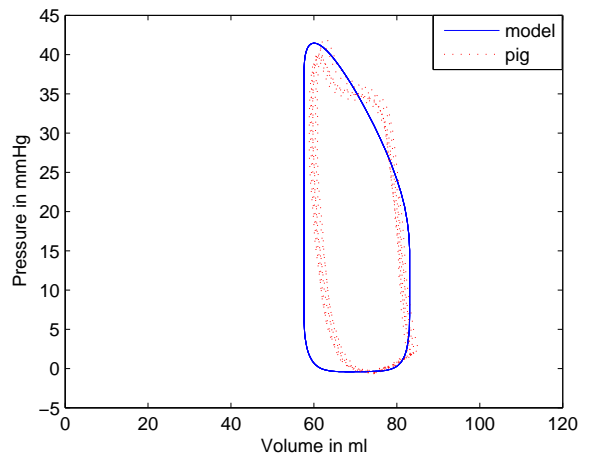

(b) 120 mins, right ventricle

Figure 20: Porcine pulmonary embolism, pig 1, Pressure-Volume Relationship 


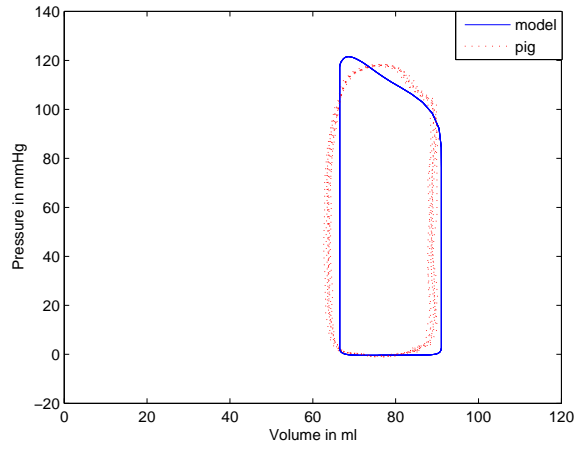

(a) 180 mins, left ventricle

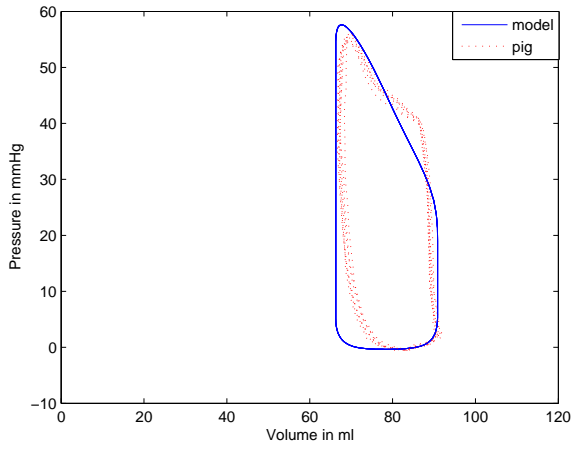

(b) 180 mins, right ventricle

Figure 21: Porcine pulmonary embolism, pig 1, Pressure-Volume Relationship
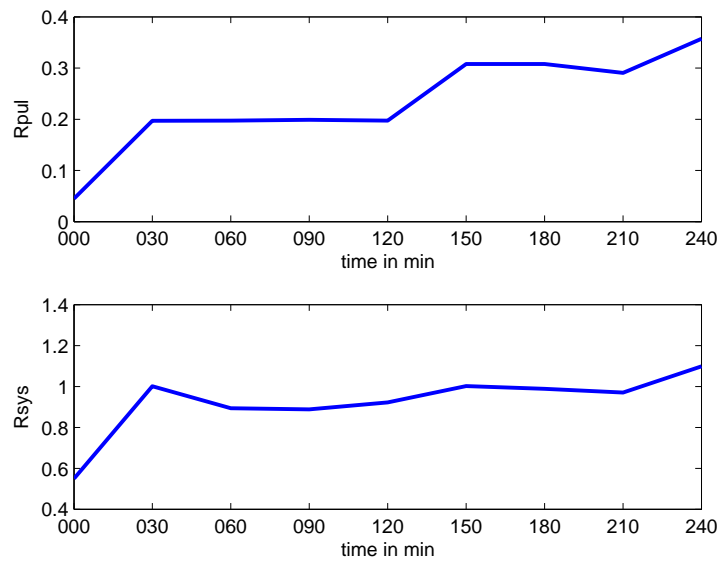

Figure 22: Porcine pulmonary embolism, pig 1: Pulmonary and vascular systemic resistance 


\begin{tabular}{|l|l|l|l|l|}
\hline \multicolumn{5}{|l|}{ Pressures $(\mathrm{mmHg})$ and Error (\%) } \\
\hline Noise & $P_{a o}$ & $P_{p a}$ & $P_{l v}$ & $P_{r v}$ \\
\hline $0 \%$ & $1.63 \pm 0.89(1.70)$ & $1.28 \pm 1.31(4.92)$ & $1.30 \pm 1.56(2.40)$ & $0.76 \pm 1.38(4.17)$ \\
$5 \%$ & $1.58 \pm 0.83(1.74)$ & $2.01 \pm 1.60(7.74)$ & $0.69 \pm 0.75(4.40)$ & $1.39 \pm 1.56(9.01)$ \\
$10 \%$ & $3.18 \pm 1.79(3.57)$ & $2.72 \pm 1.96(9.33)$ & $1.36 \pm 1.63(6.29)$ & $1.76 \pm 1.99(9.62)$ \\
\hline
\end{tabular}

\begin{tabular}{|l|l|l|}
\hline \multicolumn{3}{|c|}{ Volumes $(m l)$ and Error $(\%)$} \\
\hline Noise & $V_{r v}$ & $V_{l v}$ \\
\hline $0 \%$ & $1.72 \pm 1.36(2.46)$ & $1.80 \pm 2.08(2.46)$ \\
$5 \%$ & $2.07 \pm 1.08(3.36)$ & $2.88 \pm 2.34(3.81)$ \\
$10 \%$ & $2.50 \pm 2.44(4.64)$ & $3.81 \pm 3.38(4.70)$ \\
\hline
\end{tabular}

\begin{tabular}{|l|l|l|l|l|l|l|}
\hline \multicolumn{7}{|l|}{ Rpul $\left(\mathrm{mmHgml}^{-1}\right)$} \\
\hline Noise & $0 \mathrm{~s}$ & $35 \mathrm{~s}$ & $70 \mathrm{~s}$ & $105 \mathrm{~s}$ & $140 \mathrm{~s}$ & $175 \mathrm{~s}$ \\
\hline $0 \%$ & $0.155(0.07)$ & $0.191(5.34)$ & $0.257(1.72)$ & $0.315(7.32)$ & $0.413(6.62)$ & $0.583(1.32)$ \\
$5 \%$ & $0.142(8.06)$ & $0.198(1.40)$ & $0.252(3.81)$ & $0.323(5.06)$ & $0.381(14.04)$ & $0.483(16.16)$ \\
$10 \%$ & $0.154(0.14)$ & $0.208(2.10)$ & $0.243(7.34)$ & $0.306(10.01)$ & $0.371(16.14)$ & $0.482(16.23)$ \\
\hline True & 0.155 & 0.201 & 0.262 & 0.340 & 0.443 & 0.576 \\
\hline
\end{tabular}

Table 1: Simulated Human Pulmonary Embolism: Mean model response errors and standard deviation for combined maximum and minimum pressures and volumes. The lower portion shows the value of pulmonary resistance, $R_{\text {pul }}$, with the true simulated value for comparison. The mean percentage errors are given in brackets. 


\begin{tabular}{|l|l|l|l|l|}
\hline \multicolumn{5}{|l|}{ Pressures $(\mathrm{mmHg})$ and Volumes $(\mathrm{ml})$} \\
\hline Pig & $P_{a o}$ & $P_{p a}$ & $P_{l v}$ & $P_{r v}$ \\
\hline$\# 1$ & $3.501 \pm 3.247(4.10)$ & $2.31 \pm 1.93(8.63)$ & $1.37 \pm 1.65(3.46)$ & $2.313 \pm 1.939(5.46)$ \\
$\# 2$ & $5.042 \pm 3.161(4.59)$ & $2.394 \pm 1.827(10.17)$ & $1.604 \pm 1.179(1.90)$ & $1.264 \pm 1.337(5.23)$ \\
$\# 3$ & $2.567 \pm 2.130(2.18)$ & $2.305 \pm 1.796(2.30)$ & $2.414 \pm 2.607(2.41)$ & $0.571 \pm 0.708(2.51)$ \\
\hline
\end{tabular}

\begin{tabular}{|l|l|l|}
\hline \multicolumn{3}{|c|}{ Volumes $(m l)$ and Error $(\%)$} \\
\hline Noise & $V_{r v}$ & $V_{l v}$ \\
\hline $0 \%$ & $1.666 \pm 1.682(2.39)$ & $1.273 \pm 1.187(1.90)$ \\
$5 \%$ & $3.468 \pm 0.870(4.21)$ & $3.007 \pm 1.609(4.75)$ \\
$10 \%$ & $3.316 \pm 2.315(5.67)$ & $2.294 \pm 2.156(3.32)$ \\
\hline
\end{tabular}

\begin{tabular}{|l|l|l|l|l|l|l|}
\hline \multicolumn{6}{|c|}{ Rpul $\left(\mathrm{mmHgml}^{-1}\right)$} \\
\hline Pig & 0 min & 60 min & $90 \min$ & $120 \mathrm{~min}$ & $180 \mathrm{~min}$ & $240 \mathrm{~min}$ \\
\hline$\# 1$ & 0.044 & 0.196 & 0.198 & 0.197 & 0.307 & 0.290 \\
$\# 2$ & 0.152 & 0.305 & 0.393 & 0.387 & 0.529 & 0.466 \\
$\# 3$ & 0.138 & 0.174 & 0.215 & 0.228 & 0.405 & 0.445 \\
\hline
\end{tabular}

Table 2: Porcine Pulmonary Embolism: Mean model response errors and standard deviation for combined maximum and minimum pressures and volumes. The lower portion shows the true value of pulmonary resistance, $R_{\text {pul }}$, with the true simulated value for comparison. The mean percentage errors are given in brackets. 